\title{
Development of cleaning system installed in horizontal moving system for maintenance of high-rise building
}

\author{
$\underline{\text { Jaemyung Huh }}{ }^{1}$, Sung-Min Moon ${ }^{1}$, Sung-Won Kim ${ }^{1}$, Daehie Hong ${ }^{2 *}$ \\ ${ }^{1}$ Department of Mechanical Engineering, Korea University, Seoul, Korea \\ ${ }^{2}$ School of Mechanical Engineering, Korea University, Seoul, Korea \\ *Corresponding author (dhhong@korea.ac.kr)
}

\begin{abstract}
Purpose Nowadays, in modern cities many high-rise buildings are equipped with curtain-walls. Before the advent of robot technology, building maintenance experts maintained curtain-walls periodically. However, their work is very dangerous and there are many falls and accidents in the workplace. Because of this danger, the manual maintenance process is very expensive. In order to solve this problem, our paper suggests a full-automatic cleaning system which is installed as a built-in guide rail robot system. Method The built-in guide rail robot system consists of two moving system: a vertical and a horizontal moving system. Especially important is the horizontal moving cleaning system. There are three units in the cleaning system: a roll-brushing unit, an injection unit, and a squeezing unit. These units work in a particular order. Having two moving systems extend the cleaning area, and the vertical moving system is equipped to charge necessary supply material. Results \& Discussion The design suggested in this paper is suitable for high-rise building maintenance. Not only is this robot is automatically workable, but it also saves maintain building time. Therefore, using this robot, building maintenance is safer and more efficient than using manual labor.
\end{abstract}

Keywords: building maintenance, cleaning system, material supply

\section{INTRODUCTION}

Nowadays, the recent height of buildings in modern cities is increasingly higher. In addition, those buildings have equipped with curtain-walls for beauty. However, dust of cities makes easily façade of curtain-walls dirty.

To prevent façade from being dirty by dust, building maintenance experts should maintain it, usually once per three months. Unfortunately, their work is too dangerous, so there are many fall and crash accidents in the workplace. Because of this danger, their employment fee is too expensive and this increases expense of the manual maintenance process.

In order to solve this problem, many engineers have developed the building maintenance robots to take the place of human labor. Those robots can be classified into two categories based on direction of maintenance: rooftop gantry type ${ }^{1,2,3}$, and built-in guide rail type $e^{4,5,6}$.

The first robot maintains façade with moving vertically by hanging on the rooftop gantry. This robot is able to clean flat surface efficiently. However, on curved surface, this robot cannot be attached perfectly, and that causes poor efficiency of maintenance work at curved surface.

Unlike the first type, the second robot cleans façade with moving along the horizontal rail that is installed in building. Not only to move horizontally, but this system also is able to move vertically by combination with vertical moving system. Additionally, it can be attached more closely at curved surface than the first. By this fact, efficiency of maintenance work on curved surface is almost constant, comparing with on flat surface. That is, the built-in guide rail type is more suitable for all-area cleaning than rooftop gantry type.

In order to replace completely the human labor by maintenance robot, with suitable type for maintenance work, development of full-automation of cleaning system is also needed, because the existing maintenance robot is semi-automatic in material charging work, and cleaning work.

So, this paper deals with the full-automatic cleaning system and material charging system. The cleaning system is installed in the horizontal moving system, and is supplied with maintenance materials from the material charging system installed in the vertical

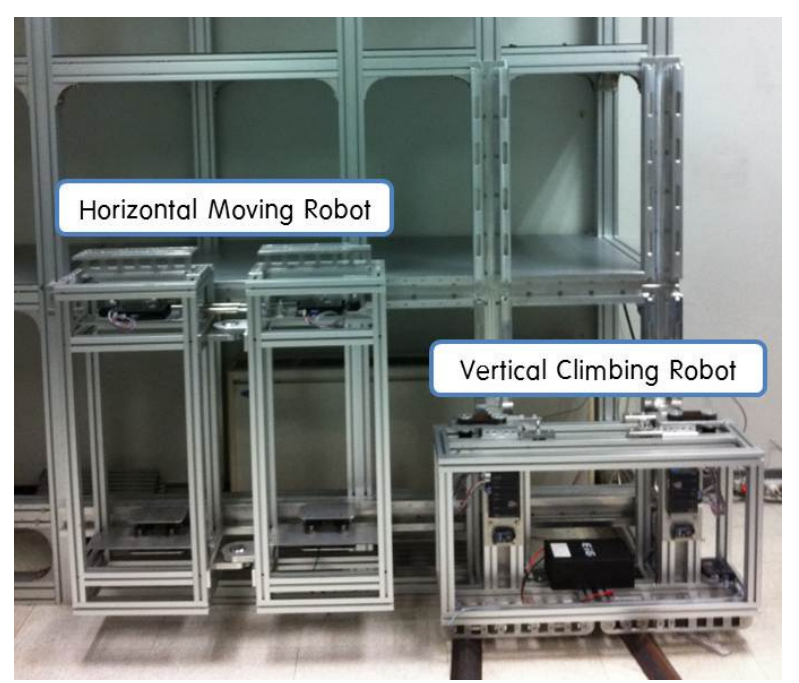

Fig.1. Building maintenance robot of the build-in guide rail type 
moving system. The overall design concept and the integrated control algorithm about the cleaning system and material charging system will be introduced. The robot used in this paper is a prototype for experiment. To determine briefly how this robot is applied in real building, this paper uses it with the test-bed building.

\section{BUILDING MAINTENANCE ROBOT BASED ON BUILT-IN GUIDE RAIL}

As mentioned previously, the building maintenance robot in this paper moves along the built-in guide rail which consists of vertical and horizontal one. As the built-in guide rail can be divided with install direction, the robot also has two systems classified by moving direction: the horizontal moving system, and the vertical moving system.

The system which do mainly maintenance work is the horizontal moving system. Its moving mechanism called wheel mechanism ${ }^{5,6}$ makes it move along the horizontal rail without separation. In the wheel mechanism, the encoders are attached to the motors, or directly to the wheels, in order to get information of location.

For smooth movement, anti-shock mechanism ${ }^{5,6}$ is installed, and it is to prevent the horizontal moving system from destroyed by shock and vibration, which is generated by installation error of rail. Moreover, for moving smoothly in curved rail, there is the curve moving mechanism ${ }^{5,6}$.

The vertical moving system uses inch-worm mechanism and hook mechanism for vertical climbing ${ }^{6}$. Especially, those two systems help to move up and down more precisely than the rooftop gantry type which uses gondola-winch system.

Using those mechanisms, the vertical moving system is to carry the horizontal moving system to the next floor and charge storage with maintenance

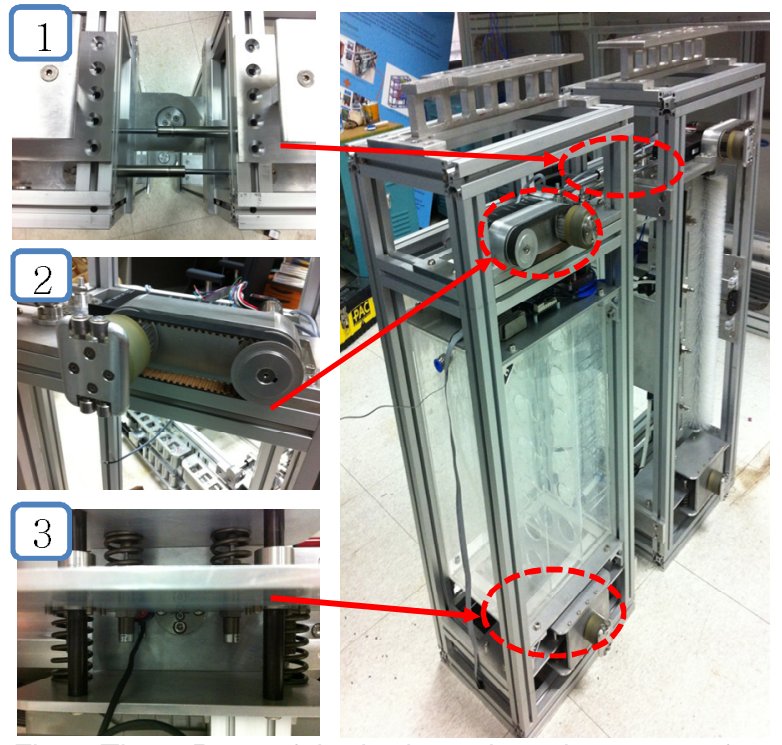

Fig.2. Three Parts of the horizontal moving system for smooth movement: 1. Curve moving mechanism, 2. Wheel mechanism, 3. Anti-shock mechanism
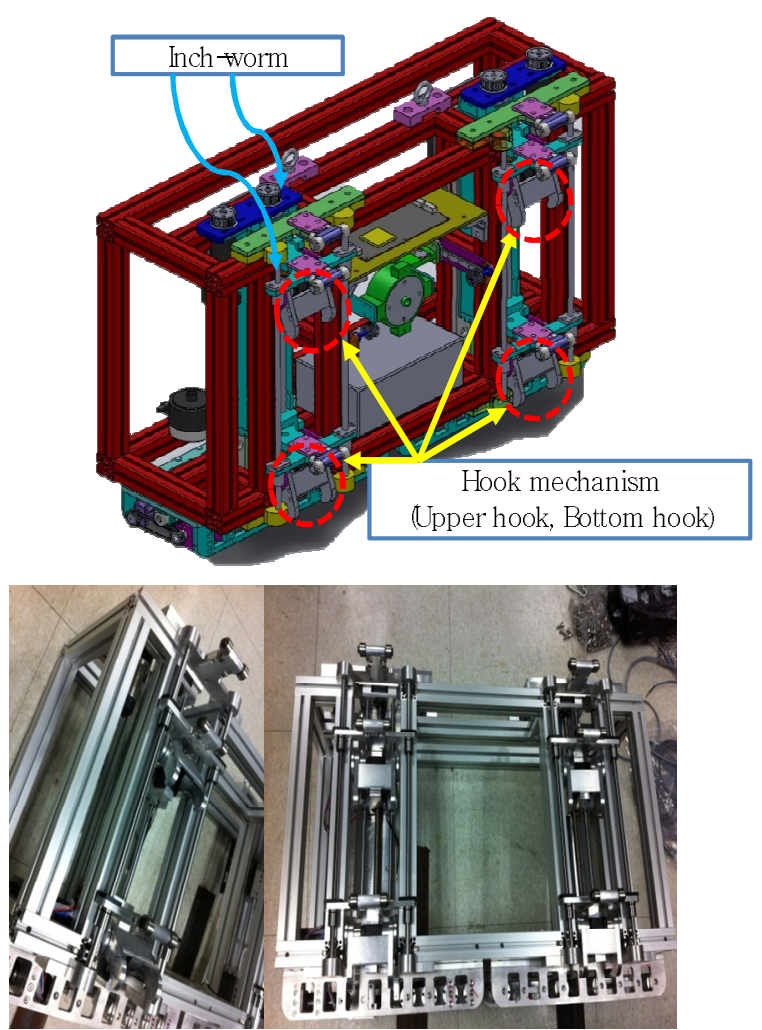

Fig.3. Hook mechanism and Inch-worm mechanism installed in the vertical moving system of the building maintenance robot

material. However, before two works, combination of two systems should be followed. For this combination, the vertical moving system of the robot has the compact docking mechanism. It is operated by only one motor. But nevertheless, it can make two motions that are docking and pulling/pushing, by using relative motion between links of it $^{6}$. When the robot comes in the next floor and the horizontal moving

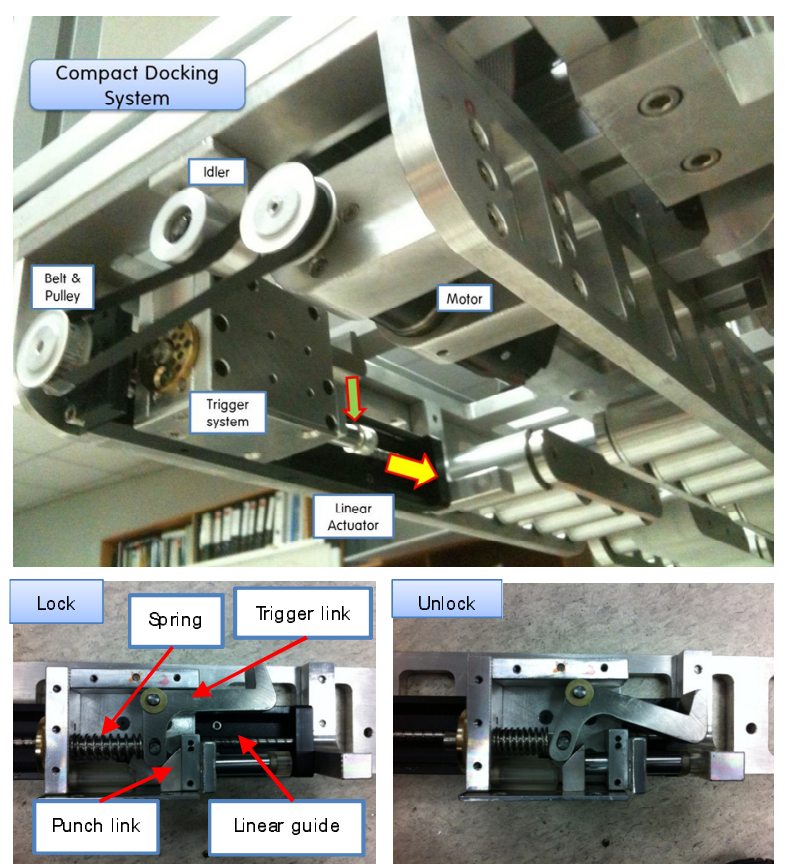

Fig.4. Configuration of the compact docking mechanism of the vertical moving system ${ }^{6}$ 
system prepares to enter the horizontal rail of that floor, the separation, whose operation order is contrary to combination, begins. In this way, the compact docking mechanism makes it possible for the vertical moving system to do two aforementioned works.

Specifically saying about how the compact docking mechanism helps to charge storage with material, proper connection between the storage and material charging system is made by exact combination of two systems.

\section{MATERIAL CHARGING SYSTEM}

For full automation of the maintenance work, charging maintenance material should be automatic. The existing maintenance robots charge material periodically by manual charging work. But, that case takes too long time, because of manual work.

So, for the robot to supply material to its storage by itself, in this paper, material charging system is developed. It has benefit that light weight of the robot is possible, because size of storage of maintenance material can be small, and save work time.

For safety, charging work should be processed with satisfying that the additional force, which is made in charging work, should not be over the required amount. Under consideration of that, this paper uses 2 stage-station of valve system. It consists of two systems that are 'Automatic-Water Dispensing System', and 'Transmitted Operation Material Supply System'. The first system is automatic and safe connection mechanism between the material source of the building and the material charging system. The second one is connection between the material charging system and the storage of the horizontal moving system.

In automatic-water dispensing system, there is linear actuator which consists of motor, pulley, and linear guide. It makes connector, which is the hose centered in steel cylinder, dock with electromagnetic fitting part by magnetic force, and be connected with electric valve of fitting part. In order to protect façade from damage in connection, the linear actuator constrains sudden strike by moving smoothly. During connection, the push-lock switches embedded in the

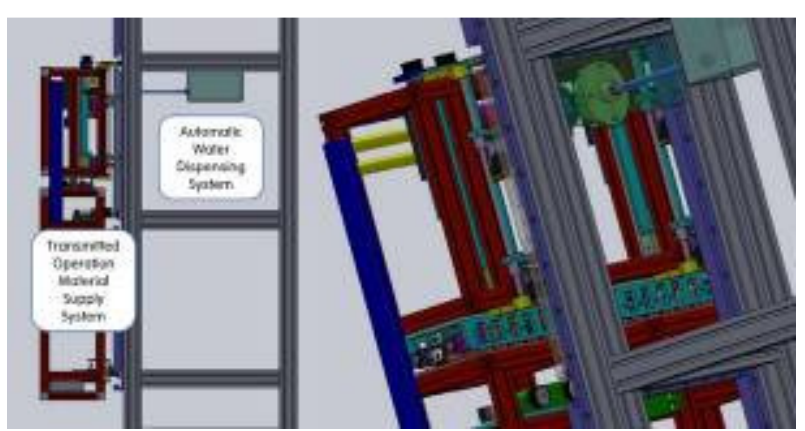

Fig.5. 2 Stage-stations of electric valve system: Automatic water dispensing system, Transmitted operation material supply system ${ }^{6}$

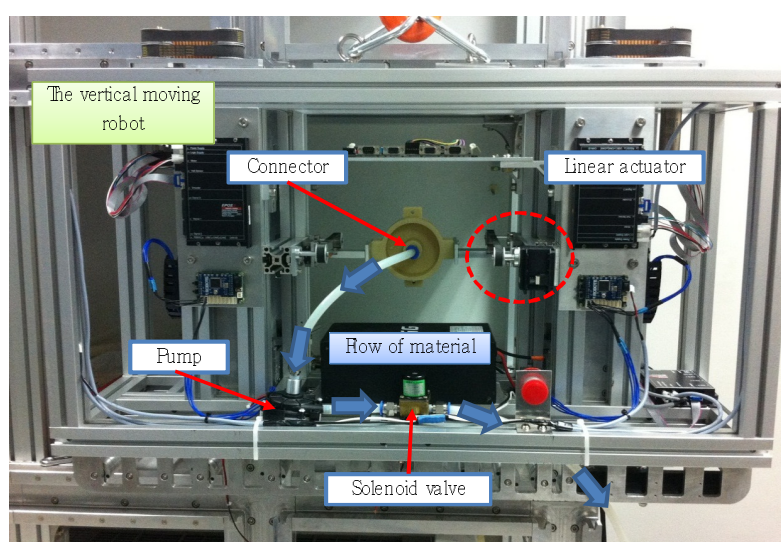

Fig.6. Configuration of the material charging system of the vertical movina svstem

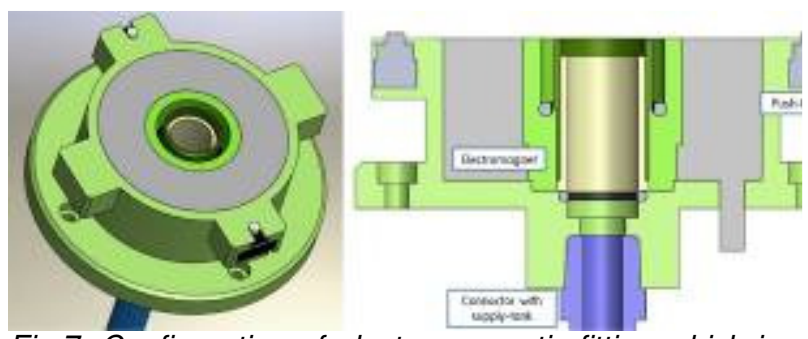

Fig.7. Configuration of electro-magnetic fitting which is installed in the building ${ }^{6}$

fitting part are pressed and pump is operated, and then electric valve is opened.

The transmitted operation material supply system is simple connection that uses only one pump and bucket. The small opened bucket is installed in the horizontal moving system, so when combination between the horizontal moving system and the vertical moving system is completed and then pump is

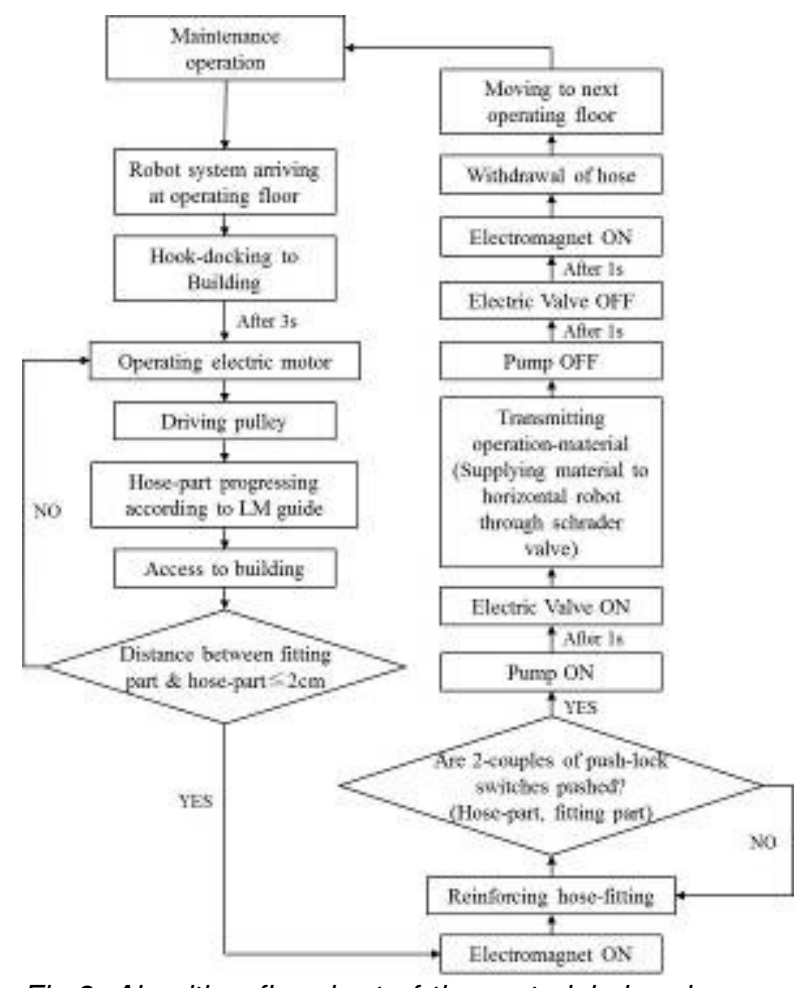

Fig.8. Algorithm flowchart of the material charging system $^{6}$ 
operated, material flow into it. The bucket is just connected with the storage, material flows again into the storage through pump.

When charging is completed, the connection between the fitting part and the connector by magnetic force is released. Next, the pump loses power, and the electric valve is closed.

All those processes are presented in algorithm flowchart of Fig.8.

\section{Cleaning system}

After supplied from the material charging system to the storage, the cleaning material is stored temporarily in the storage, and then flows to the cleaning system through pump, when the cleaning system starts maintenance work.

The cleaning system is only part that is able to do maintenance work in this robot. So, it should be designed to optimize efficiency of maintenance work. For that case, using simple maintenance mechanism, it saves electric energy by using few actuators. Also, it can simultaneously take care of moving along the rail and maintaining the façade. Thanks to that, time for the maintenance work can be less than conventional method.

The cleaning system consists of three units: Rollbrushing unit, Injection unit, and Squeezing unit. Those units are aligned horizontally, and installed in order which is determined by order of usual cleaning work.

\section{Roll-Brushing unit}

The roll-brushing unit accesses the target cleaning area firstly. In usual cleaning work, sweeping is the first process that is to remove bigger dust before wiping. Roll-brushing unit is to sweep bigger dust attached at the façade of building. Its sweeping type is rotation-sweeping that is operated by motor. In addition, size of the roll-brushing unit, especially about diameter of roll brush, is determined to not

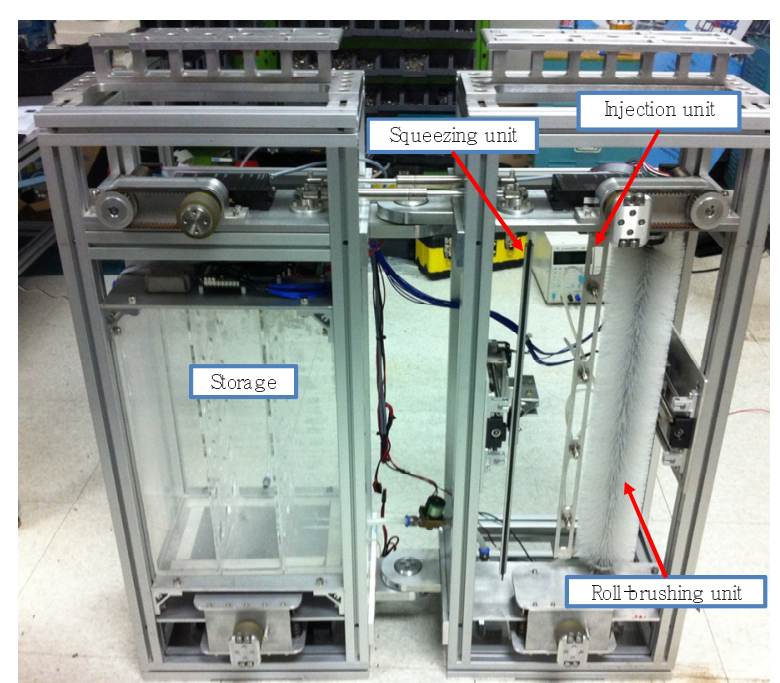

Fig.9. Three units of the cleaning system and the storage

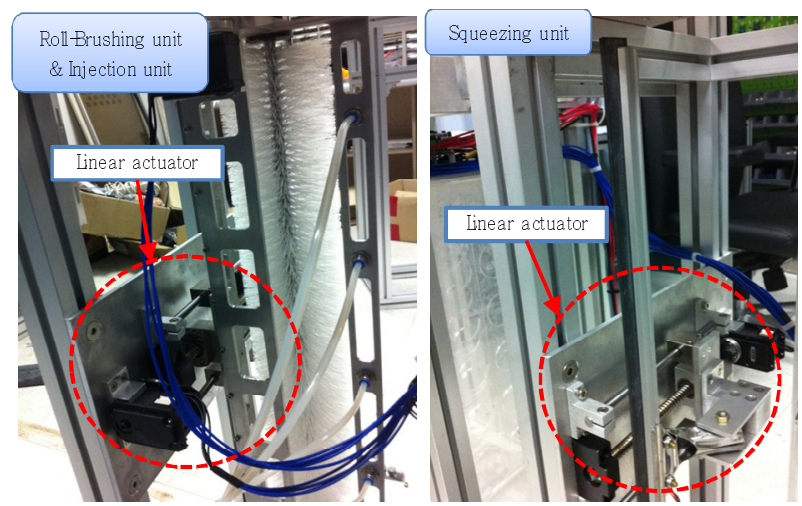

Fig.10. Motion of three units is made by linear actuator which consists of motor and linear guide

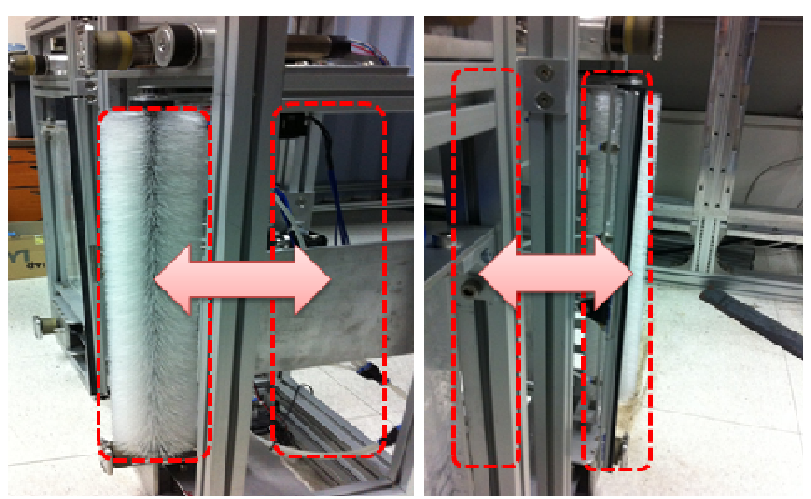

Fig.11. Three units can be moved inside or outside, by linear actuators

intercept nozzle's injection range.

\section{Injection unit}

After the roll-brushing unit sweeps target cleaning area, the injection unit accesses it secondly. Nozzles of this unit are supplied with maintenance material in storage, and inject it to the façade. This unit is assembled with the roll-brushing unit, so if it comes close to the obstacles like mullion, it will go inside the horizontal moving system with the roll-brushing unit. The reason why it is connected with the roll-brushing unit is that the roll-brushing unit is to not intercept nozzle's range of injection.

\section{Squeezing unit}

The injected water separates the small dust or stain which is not removed by the roll-brushing unit. The squeezing unit removes water that is polluted by small dust and stain.

In order to do that without collision, It also has linear motion that move inside and outside the robot, by linear actuator, which consists of motor and linear guide. Like the preceding two units, it is able to avoid crash with obstacles, by moving inside the robot. On the contrary to that, with moving outside the robot, it can remove perfectly dirty water by rubbing the facade with pressing properly it. 


\section{INTEGRATED CONTROL ALGORITHM}

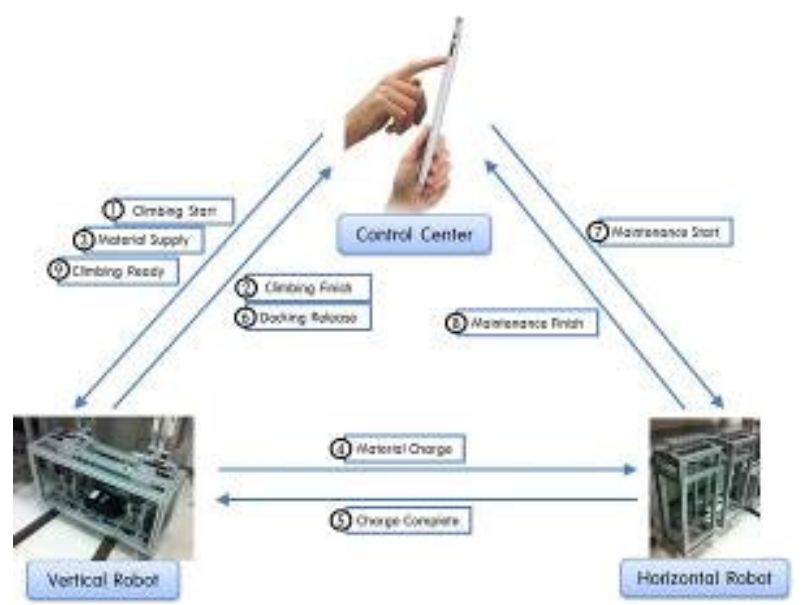

Fig.12. Control process of the building maintenance robot $^{6}$

For all part of the robot to co-operate harmoniously, control system should give the instruction to each part through control-center. Desired instruction for maintenance work is transferred to the vertical moving system through the wireless modem from the control center.

First, the instruction transferred to the vertical moving system is about its vertical climbing. It carries the horizontal moving system to target floor for maintenance. When the vertical moving system arrives at target floor, control-center gives the vertical moving system an instruction to operate material charging system.

After the instruction is transferred to the control system of the vertical moving system, the control system transfers signal for linear actuator of the material charging system to have the connector approach the electromagnetic fitting part. When connection with material source is completed, the control system orders pump and solenoid valve to operate. So, when the solenoid valve opens, the pump gives force for material to flow into the storage.

When the storage of the horizontal moving system is full of material, flow-meter and timer give information about that to the control center. After that, combination by the docking system is released by docking release signal of control center. Then, the control center orders the horizontal moving system to start maintenance work. While maintenance work in process, the pump and solenoid valve which are installed in the horizontal moving system operate for material in storage to flow to nozzles of the injection unit.

In the maintenance work, when the contact sensor detects obstacles like mullion, information of the contact sensor is delivered to the control board which is installed in the horizontal moving system. And then, the control board orders that linear actuators have the units of the cleaning system move inside.

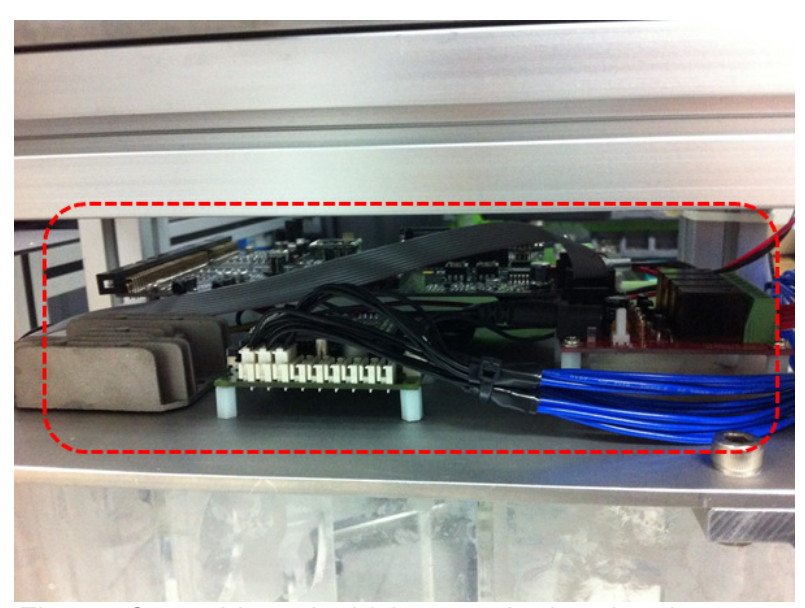

Fig.13. Control board which controls the cleaning system

Specifically saying about avoidance process, when the roll-brushing unit nears obstacle, the rollbrushing unit and the injection unit move inside, while nozzles of the injection unit keep injecting material to the facade. When the roll brushing unit pass exactly obstacle, two units move outside, in order to sweep small area near obstacle. Although the injection unit doesn't pass obstacle completely in that time, there is no smash between façade and injection unit. The reason is that the injection unit is designed more inward than the roll brushing unit. Meanwhile, the squeezing unit also conducts same process.

When the maintenance work is completed, encoders give distance information to the control center, and then control center confirm that the horizontal moving system arrives at the end, by calculating position of the robot with distance information. The control center, then, sends a return order to it. Finally nextfloor transportation work is carried on.

By repeating these processes, the maintenance robot is able to maintenance all area of the building.

\section{Conclusion}

This paper describes a new type of the building maintenance robot system whose movement is based on the built-in guide rail type, and deals with the material maintenance and the cleaning system.

By the wheel mechanism, the curve moving mechanism, and the anti-shock mechanism, the horizontal moving system can move smoothly along the horizontal rail and also the curved rail without damage. Not only moving horizontally, but moving vertically is also possible by combination between the horizontal moving system and the vertical moving system. The combination can be made by compact docking system. Additionally, in the vertical moving system, it has the inch-worm system using hook mechanism, in order to realize precise vertical climbing.

For stable maintenance work, material supplying is to be stable. So, the robot uses the material charging system. In order to gain high efficiency of mainte- 
nance work, the cleaning system is developed into simple mechanism.

Furthermore, in order to apply perfectly this robot's mechanism to the workplace, detail design should be made and data of damage caused by external force also should be gained. This prototype robot will answer how those works will be conducted.

\section{ACKNOWLEDGMENT}

The work presented in this paper was funded by BMRC(Building-Façade Maintenance Robot Research Center), supported by Korea Institute of Construction and Transportation Technology Evaluation and Planning(KICTEP) under the Ministry of Land, Transport and Maritime Affairs(MLTM).

\section{References}

1. Akinfiev, T., Armada, M., Nabulsi, S., "Climbing cleaning robot for vertical surfaces", Industrial Robot: An International Journal, Vol. 36(4), pp. 352357, 2009.

2. Gambao, E., Hernando, M., "Control System for a Semi-automatic Façade Cleaning Robot", ISARC Int. Symp on Automation and Robotics in Construction, pp. 406-411, 2009.

3. Elkmann, N., Kunst, D., Krueger, T., Lucke, M., Böhme, T., Felsch, T., Stürze, T., "SIRIUSc - Facade Cleaning Robot for a High-Rise Building in Munich, Germany", Climbing and Walking Robots, Part IX, pp. 1033-1040, 2005.

4. Zhang, H., Wang, W., Liu, R., Zhang, J., Zong, G., "Locomotion Realization of an Autonomous Climbing Robot for Elliptic Half-shell Cleaning", Industrial Electronics and Applications 2007 ICIEA $20072^{\text {nd }}$ IEEE Conference, pp.1220-1225, 2007.

5. Moon, S.M., Kim, S.W., Park, S.R., Hong, D.H., "Development of building-façade maintenance robot with docking station based on vertical climbing mechanism", ISARC Int. Symp. on Automation and Robotics in Construction, Vol. S34-S36, pp.12211222, 2011.

6. Moon, S.M., Hong, D., Kim, S.W., Park, S., "Building Wall Maintenance Robot Based on Built-in Guide Rail", Proc. of 2012 IEEE International Conference on Industrial Technology, pp. 509-514, 2012. 